\title{
Characterization of autism spectrum disorder and neurodevelopmental profiles in youth with $\mathrm{XYY}$ syndrome
}

Lisa Joseph ${ }^{1}$, Cristan Farmer ${ }^{1}$, Colby Chlebowski ${ }^{1}$, Laura Henry ${ }^{1}$, Ari Fish², Catherine Mankiw², Anastasia Xenophontos², Liv Clasen², Bethany Sauls', Jakob Seidlitz², Jonathan Blumenthal², Erin Torres², Audrey Thurm ${ }^{1 \dagger}$ and Armin Raznahan ${ }^{2 *}$

\begin{abstract}
Background: XYY syndrome is a sex chromosome aneuploidy that occurs in 1/850 male births and is associated with increased risk for neurodevelopmental difficulties. However, the profile of neurodevelopmental impairments, including symptoms of autism spectrum disorder (ASD) in XYY remains poorly understood. This gap in knowledge has persisted in part due to lack of access to patient cohorts with dense and homogeneous phenotypic data.

Methods: We evaluated a single-center cohort of 64 individuals with XYY aged 5-25 years, using a standardized battery of cognitive and behavioral assessments spanning developmental milestones, IQ, adaptive behavior, academic achievement, behavioral problems, and gold-standard diagnostic instruments for ASD. Our goals were to (i) detail the neurodevelopmental profile of XYY with a focus on ASD diagnostic rates and symptom profiles, (ii) screen phenotypes for potential ascertainment bias effects by contrasting pre- vs. postnatally diagnosed XYY subgroups, and (iii) define major modules of phenotypic variation using graph-theoretical analysis.
\end{abstract}

Results: Although there was marked inter-individual variability, the average profile was characterized by some degree of developmental delay, and decreased IQ and adaptive behavior. Impairments were most pronounced for language and socio-communicative functioning. The rate of ASD was 14\%, and these individuals exhibited autism symptom profiles resembling those observed in ASD without XYY. Most neurodevelopmental dimensions showed milder impairment among pre- vs. postnatally diagnosed individuals, with clinically meaningful differences in verbal IQ. Feature network analysis revealed three reliably separable modules comprising (i) cognition and academic achievement, (ii) broad domain psychopathology and adaptive behavior, and (iii) ASD-related features.

Conclusions: By adding granularity to our understanding of neurodevelopmental difficulties in XYY, these findings assist targeted clinical assessment of newly identified cases, motivate greater provision of specialized multidisciplinary support, and inform future efforts to integrate behavioral phenotypes in XYY with neurobiology.

Trial registrations: ClinicalTrials.gov NCT00001246, "89-M-0006: Brain Imaging of Childhood Onset Psychiatric Disorders, Endocrine Disorders and Healthy Controls."

Keywords: Sex chromosome aneuploidies, Autism spectrum disorder symptoms, Learning disabilities, Adaptive behavior, Cognitive functioning

\footnotetext{
* Correspondence: raznahana@mail.nih.gov

${ }^{+}$Audrey Thurm and Armin Raznahan contributed equally to this work.

${ }^{2}$ Developmental Neurogenomics Unit, Human Genetics Branch, National

Institute of Mental Health, National Institutes of Health, Bethesda, MD, USA

Full list of author information is available at the end of the article
}

(c) The Author(s). 2018 Open Access This article is distributed under the terms of the Creative Commons Attribution 4.0 International License (http://creativecommons.org/licenses/by/4.0/), which permits unrestricted use, distribution, and reproduction in any medium, provided you give appropriate credit to the original author(s) and the source, provide a link to the Creative Commons license, and indicate if changes were made. The Creative Commons Public Domain Dedication waiver (http://creativecommons.org/publicdomain/zero/1.0/) applies to the data made available in this article, unless otherwise stated. 


\section{Background}

Sex chromosome aneuploidy is the carriage of sex chromosome complements other than XX in females or $\mathrm{XY}$ in males, and is estimated to occur in $1 / 420$ live births [1]. One of the most common is XYY syndrome, with an estimated prevalence of $\sim 1 / 850$ male births [1]. Longstanding interest and controversy regarding the behavioral phenotype of XYY was sparked by an early series of influential yet methodologically flawed casecontrol studies suggesting an association between XYY and commission of violent crimes [2]. More rigorous research in larger cohorts has robustly refuted this association [3, 4], further noting that individuals with XYY may be at increased risk for a range of neurodevelopmental difficulties [4-8].

To date, the neurodevelopmental phenotype of XYY has been most consistently associated with lowered intelligence quotient (IQ), language impairments, academic problems, and difficulties with attention and social interaction [9]. Studies of general cognitive ability in XYY report IQ deficits of approximately 10 points [4, 10], preferentially affecting verbal domains [4]. Relatedly, language delay $[10,11]$ and subsequent language impairments are consistently reported in both birth and clinical cohorts $[4,6]$. The increased rates of academic difficulties and special education needs $[4,6$, $12,13]$ are most pronounced in the reading domain $[12,13]$. XYY has also been associated with impairments in adaptive functioning, potentially exceeding that predicted by lowered IQ alone [7]. Consistent with the documented reductions in IQ and adaptive functioning, rates of intellectual disability are increased relative to the general population [8], as are other neurodevelopmental disorders including attention deficit hyperactivity disorder [8] and autism spectrum disorder (ASD) [7, 14].

The potential association between XYY and ASD has undergone intense study $[6,7,14-16]$, motivated in part by an unbalanced sex ratio of ASD among karyotypically normal children who are diagnosed with ASD [17]. The most methodologically rigorous study yielded an ASD diagnostic rate of $38 \%$ in 57 participants [7]. However, it is not known whether the phenotypic presentation of ASD in youth with XYY is distinguishable from those with idiopathic ASD, and whether ASD-related features reliably segregate with other phenotypic aspects of XYY.

Here, we examine the neurodevelopmental phenotype of XYY in depth within a previously unpublished cohort of 64 youth aged 5-25 years, representing the largest single-center study of XYY. Our study was designed to build upon existing knowledge regarding neurodevelopment in XYY in three key directions.

First, there are no existing studies that contemporaneously capture the full range of neurodevelopmental dimensions using homogenous single-center protocols with a common set of instruments across all participants. We sought to achieve dense and homogenous phenotypic characterization within a large cohort to detail multiple developmental dimensions in XYY, and systematically examine inter-relationships between different aspects of the neurodevelopmental profile.

Second, although elevated rates of ASD in XYY relative to the general population are noted in independent reports [6, 7], studies have not uniformly applied a gold-standard ASD diagnostic battery for all participants. Thus, we address this need by focusing on updating the estimate of the ASD diagnostic rate in groups identified with XYY, qualitatively comparing the profile of ASD features in XYY to ASD without XYY and capturing relationships between ASD and other developmental phenotypes. To meet these goals, we gathered a diverse set of ASD-related measures that can support research-level diagnostic assessment (i.e., Autism Diagnostic Observation Schedule, second edition (ADOS-2; [18]), Autism Diagnostic Interview, Revised (ADI-R; [19]) and provide complementary dimensional measures of ASD-related traits (i.e., Social Responsiveness Scale, second edition (SRS-2; [20]), Social Communication Questionnaire (SCQ; [21]) and Repetitive Behavior Scale, Revised (RBS-R; [22]). Finally, the potential for ascertainment bias has continued to pose a major challenge for studies of neurodevelopment in XYY, given the likely low detection rate and the fact that neurodevelopmental difficulties often precipitate testing in postnatally diagnosed cases [23]. Here, we harness differences between preand postnatally identified XYY subgroups $[5,15]$ as a proxy test for potential ascertainment bias effects.

\section{Methods}

\section{Participants}

Singleton males $(N=64)$ aged 5 to 25 years with XYY were enrolled in a phenotypic characterization study at the National Institutes of Health (NIH) (Table 1). Informed consent and assent was obtained from all participants and their parents; all study procedures were approved by an NIH Institutional Review Board. Participants were recruited through the Association for $\mathrm{X}$ and Y Chromosome Variations (AXYS; genetic.org) and the NIH Clinical Center Office of Patient Recruitment. XYY was confirmed and mosaicism ruled out by karyotype testing of a minimum of 50 metaphases, either through the study or confirmed from community karyotype reports when blood draw was not possible.

\section{Measures}

\section{Developmental history}

Timing of developmental milestones (i.e., first words, first use of phrases, independent walking, continence) 
Table 1 Participant characteristics by time of diagnosis

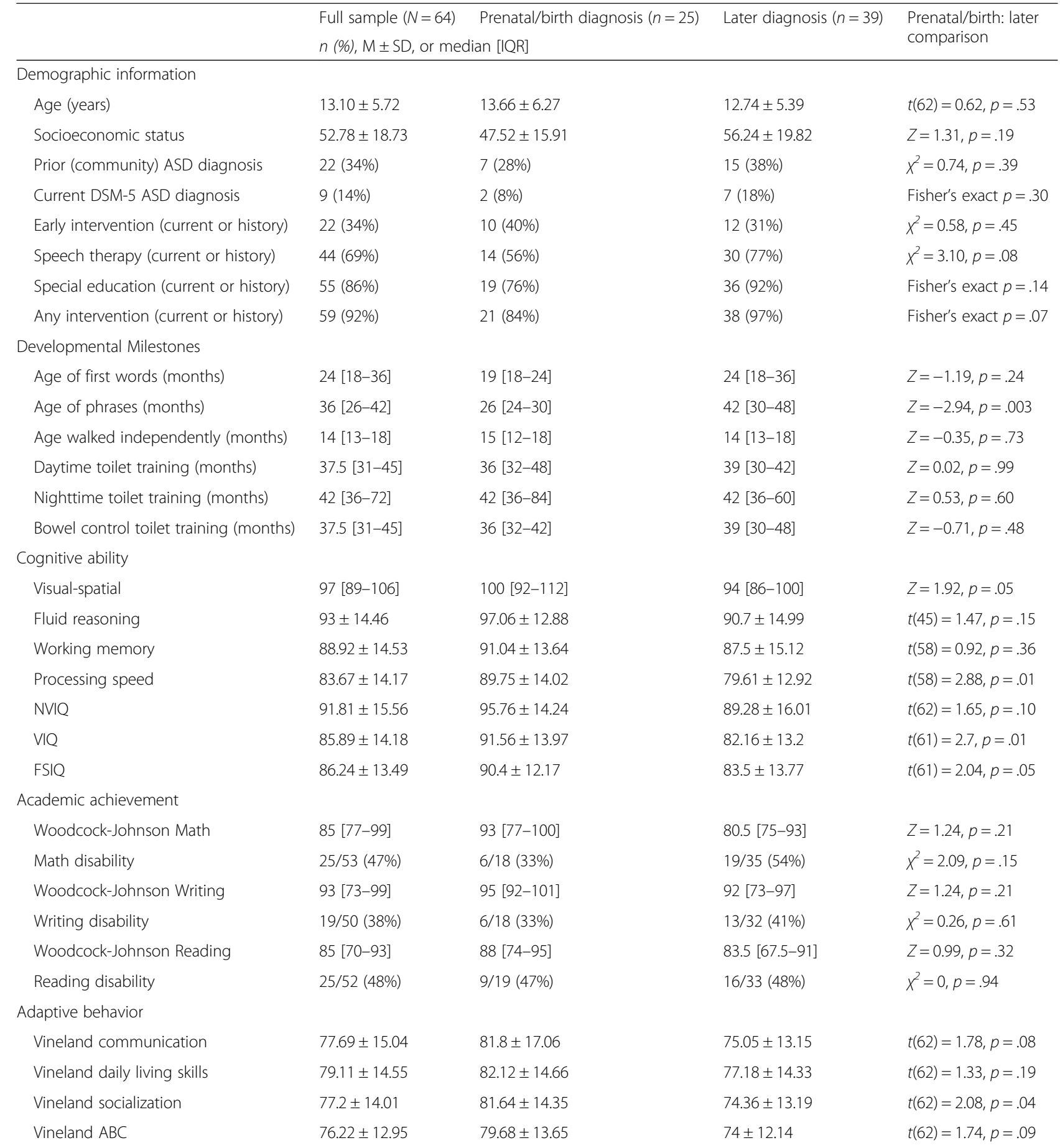


Table 1 Participant characteristics by time of diagnosis (Continued)

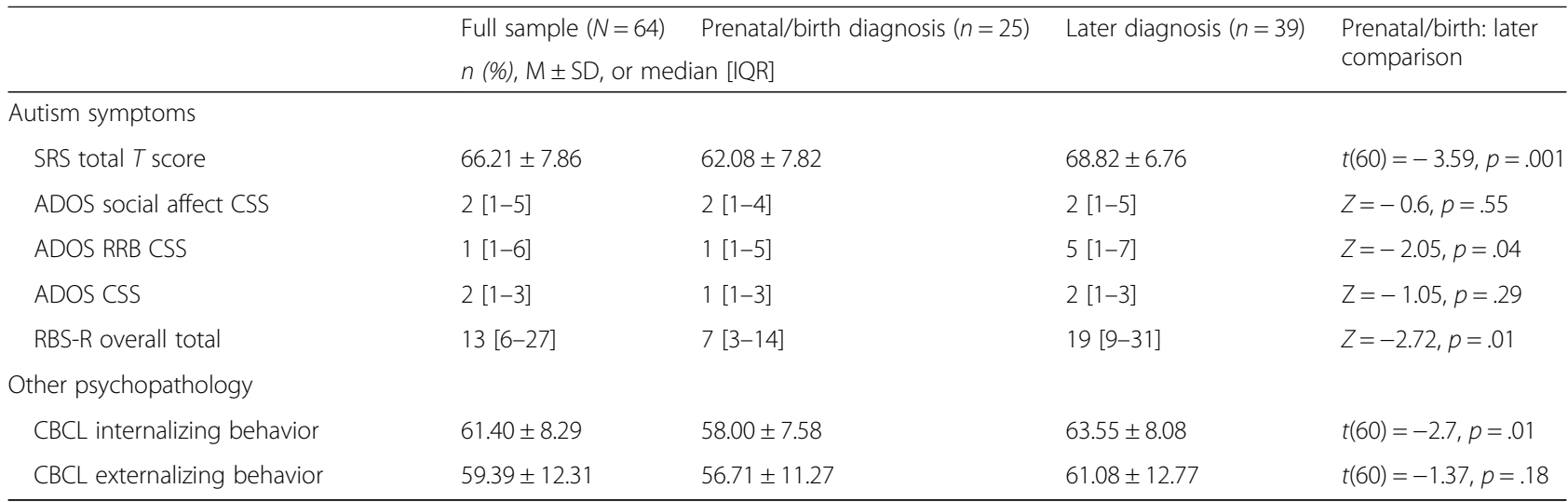

Variables that were not normally distributed (significant Shapiro-Wilk statistic) are described with median and interquartile range and tested using the Wilcoxon rank-sum test. Normally distributed variables are described with mean and standard deviation and tested using an independent samples $t$ test (where DF have decimals, Satterthwaite approximation was used)

was obtained using the ADI-R [19], which also queried intervention services and existing neurodevelopmental diagnoses. The timing of XYY diagnosis (either prenatal/ birth or postnatal) was based on parent report.

\section{Socioeconomic status}

The Hollingshead two-factor index was used to assess socioeconomic status of participants, with education and occupation factors included.

\section{Cognitive ability}

The Wechsler Preschool and Primary Scale of Intelligence, fourth edition, Wechsler Intelligence Scale for Children, fifth edition, or Wechsler Adult Intelligence Scale, fourth edition was used to assess intelligence. If the participant had been tested with a Wechsler scale within 1 year $(n=4)$, the Wechsler Abbreviated Scale of Intelligence, second edition was used.

\section{Adaptive function}

The Vineland Adaptive Behavior Scales, second edition (VABS; [24]) is a standardized semi-structured caregiver interview to assess adaptive function in the domains of communication, daily living skills, socialization, and motor skills.

\section{Academic achievement}

The Woodcock-Johnson Tests of Achievement, fourth edition (WJ-IV) is a psychoeducational assessment of academic achievement.

\section{Neurodevelopmental and behavioral phenotyping}

The ASD diagnostic battery had three components: the ADOS-2, the ADI-R, and consensus of at least two clinicians in completing the DSM-5 diagnostic criteria checklist [25]. Assessments were performed by licensed clinical psychologists (L.J., A.T., C.C.) with extensive
ASD evaluation experience, who met research reliability standards on the ADI-R and ADOS-2.

Caregiver-rated screening questionnaires were also used to assess ASD-related symptoms. These included the SRS-2, the SCQ, and the RBS-R. Other behavioral problems were assessed using either the Child Behavior Checklist or the Adult Behavior Checklist, depending on the age of the participant (referred to collectively as CBCL).

\section{Statistical analyses}

Variables were assessed for normality prior to analysis; group differences for those with significant Shapiro-Wilk statistics were analyzed with a nonparametric alternative (Wilcoxon rank sum). Normally, distributed variables were assessed using $t$ tests (independent samples for comparisons between participants diagnosed prenatally vs. at birth, or single-sample for comparison to population norms), with Satterthwaite-adjusted values in the presence of unequal variance.

To facilitate graphical comparison of the XYY sample to the ASD population, scores were $Z$ normalized against ASD normative data drawn from the Simons Simplex Collection (SSC), a research cohort ascertained primarily from autism clinics with gold-standard diagnostic measures used to confirm diagnoses; we restricted this sample to include only males $(N=1877)$. For consistency with the SSC ASD data, the XYY sample was restricted to include participants younger than 18 years $(n=48)$.

Psychometric properties were quantified for the ASD scales. Sensitivity and specificity, as well as the area under the curve (AUC) were calculated against DSM-5 ASD diagnosis. These values were also calculated for participants with and without behavior problems, as indicated by CBCL internalizing or externalizing $T$ scores greater than or equal to 64 . 
The inter-relationships among phenotypic variables in $\mathrm{XYY}$ were examined as follows. Any variables with a scaled mean correlation with other variables of less than - 2 were removed (SRS-2 awareness score, and age at walking independently). Matrices of pairwise Pearson correlations for the remaining variables were generated, using 1000 separate bootstrap draws of 64 individuals (with replacement). Each matrix was submitted to hierarchical clustering, using the gap statistic method [26]. A single square adjacency matrix was constructed using the proportion of times variable pairs were co-clustered across all 1000 analyses. Finally, a network representation of this adjacency matrix was used to define modules of phenotypic variables based on the consensus of 1000 runs of the Louvain algorithm in the MATLAB Brain Connectivity Toolbox. The Louvain algorithm gamma value was set at 1.2 by defining the local minimum of the global mean nodal versatility curve [27].

Alpha was set to .05 to reflect the descriptive nature of this report. Analyses were performed in SAS/STAT Version 9.3 and R 3.3.0 [28]; graphics were created using the igraph [27], superheat [29], and ggridges [30] packages in R 3.3.0.

\section{Results}

The sample ranged in age from 5 to 25 years and was predominantly white $(n=58,91 \%)$. The majority $(n=39$, $61 \%)$ received their XYY diagnosis sometime after birth (mean age of diagnosis $=6.08 \pm 4.55$, range 0.02 years to 16.48 years).

Table 1 and Fig. 1 summarize ratings across all neurodevelopmental domains examined in our cohort. Impairments were greatest for language and socio-communicative functioning, and least for math and non-verbal domains (Fig. 1a). Individual domains are considered separately below.

\section{Early development and intervention history}

Using a threshold of 24 months [31], we observed delays in the median age of single word acquisition, phrase speech, and independent walking. Median ages for continence milestones were in the fourth and fifth years, constituting delay [32]. Those who were diagnosed prenatally did not differ from those diagnosed later in age of first words, but their median age of phrase speech was about 16 months earlier (see Table 1; Fig. 1b). The groups did not differ on other milestones, walking, daytime continence, nighttime continence, and bowel continence. By parent report, nearly all participants had received some sort of intervention during childhood (see Table 1), though only about one-third received some type of intervention service prior to the age of 3 years.

\section{Cognitive profile}

One participant, whose first language was not English, received only the non-verbal battery. Among the remaining 63 participants, full-scale IQs (FSIQ) ranged from 53 to $112(n=34,54 \%$ in the average range, $n=22$, $35 \%$ in the borderline range, $n=6,9 \%$ in the mild range, and $n=1,2 \%$ in the moderate range). Six participants met DSM-5 criteria for intellectual disability. The average scores for nonverbal IQ (NVIQ) and verbal IQ (VIQ) were in the low-average range, both significantly different from the population mean (non-verbal: $t(63)=$ 4.21, $p=<.0001$; verbal: $t(62)=7.90, p<.0001$ ) (see Fig. 1a). The non-verbal/verbal split was statistically significant $(5.79 \pm 12.75, t(62)=3.61, p=.0006)$. Mean cognitive scores for participants diagnosed prenatally/at birth were higher than for those diagnosed later; differences were statistically significant only for the processing speed subscale and VIQ (Table 1).

\section{Academic achievement}

Mean Woodcock-Johnson scores were significantly lower than the population average: Reading, $(t(49)=-8.66, p$ $<.0001)$, mathematics $(t(48)=-6.77, p<.0001)$, and writing $(t(45)=-4.54, p<.0001)$ (see Fig. 1c). Per the DSM-5, criteria of at least one standard score below 78 (1.5 SD below population mean), and an FSIQ above 70 are used to indicate a specific learning disability (SLD); 63\% $(n=37)$ met for at least one SLD. There was no significant difference in the rate of any SLD between participants diagnosed

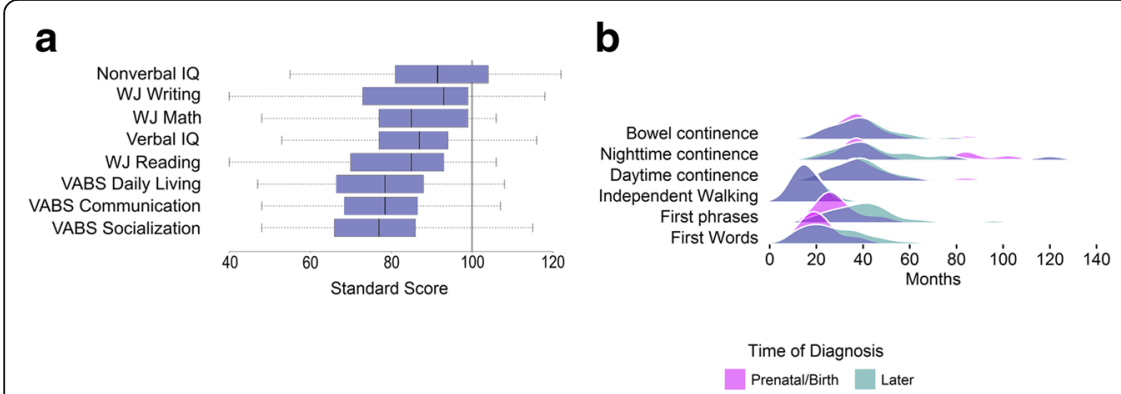

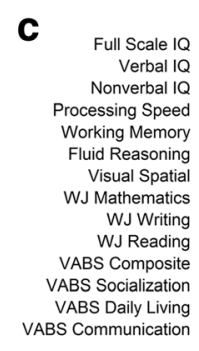

Prenatal/Birth La

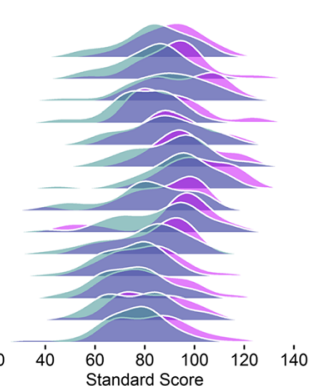

Fig. 1 Phenotypic profiles. a All differences from the population mean of 100 are statistically significant, $p<.0001$. b Milestones. Two participants with extreme continence data (> 140 months) are not represented. c Neurodevelopmental phenotype 
prenatally/at birth or postnatally $\left(\chi^{2}(1)=0.013, p=.91\right)$, nor were there differences in Woodcock-Johnson subscale scores (see Table 1).

\section{Adaptive behavior}

The VABS-II Adaptive Behavior Composite scores ranged from 42 to 112, and were significantly lower than the population average $(t(63)=14.69, p<.0001)$. The adaptive behavior profile of the sample was relatively flat (see Fig. 1c). Socialization scores in participants diagnosed with XYY after birth were significantly lower than in participants diagnosed prenatally/at birth; communication and daily living skills did not differ between groups (see Table 1). The Adaptive Behavior Composite was significantly lower than FSIQ $(t(62)=6.31, p<.0001)$, and older participants tended to have more impaired Adaptive Behavior Composite scores $(r=-0.55, p=.01)$.

\section{Autism spectrum disorder Diagnostic rates}

Twenty-two (33\%) participants carried a community diagnosis of ASD. Among the 63 participants who received ASD diagnostic measures in this study, nine (14\%) participants also met criteria for a DSM-5 diagnosis of ASD. Participants with and without DSM-5 ASD did not differ in age $(t(61)=-0.45, p=.66)$ or FSIQ $(t(60)=0.47, p=.64)$, although Adaptive Behavior Composite scores were lower in those with ASD (67.22 \pm 7.90 vs. $77.69 \pm 13.19 ; t(61)=2.30, p=.03)$.

\section{Dimensional ratings}

The median ADOS calibrated severity score was in the unaffected range (see Table 1). SRS-2 total $T$ scores in this sample ranged from 50 to 89 , and the mean cohort $T$ score was significantly higher than the population mean of $50(t(61)=16.24, p<.0001)$.

\section{Timing of XYY diagnosis and ASD}

Although the rate of DSM-5 ASD diagnosis did not differ by timing of XYY diagnosis (Table 1), participants diagnosed postnatally had significantly higher SRS-2 scores, ADOS-2 Restricted/Repetitive Behavior severity scores and RBS-R Total Scores (see Table 1).

\section{Sensitivity and specificity of ASD measures}

The sensitivity and specificity of the diagnostic instruments (ADOS-2 and ADI-R) were high (Table 2). These psychometric profiles of the screening instruments (SRS-2 and SCQ) were more variable; the SRS-2 demonstrated excellent sensitivity and poor specificity, while the SCQ had moderate levels of both.

While both the sensitivity and specificity of the ADI-R were robust to the influence of additional psychopathology (measured by CBCL internalizing and externalizing), the sensitivity of the ADOS-2 was affected by high levels of externalizing behaviors (Table 2). In contrast, the specificity of the screening measures (SRS-2 and SCQ) was particularly low among participants who had clinically significant levels of internalizing or externalizing symptoms.

\section{Descriptive comparison of $X Y Y$ and youth with ASD}

The scores from the XYY sample were $Z$ normalized against a large sample of males with ASD (Fig. 2 a, b). Generally, the profile of children with XYY and DSM-5 ASD did not deviate from that observed in the SSC ASD sample. As expected, the profile of ASD symptoms was generally more severe among participants with DSM-5 ASD than the mean profile in subgroups without ASD or with only a community diagnosis (Fig. 2c).

\section{Integrative analysis of neurodevelopmental features in XYY}

Graph-theoretical analysis of the inter-relationship among the phenotypic variables suggested three separable sub-sets of neurodevelopmental features (Fig. 3): (i) cognition and academic achievement, (ii) broad domain psychopathology and adaptive behavior, and (iii) ASDrelated features. Thus, adaptive functioning across individuals with $\mathrm{XYY}$ appeared to be more closely related to broad-domain psychopathology (especially internalizing symptoms vs. externalizing symptoms) than to cognitive ability. Network visualization also suggested that the cognitive and ASD-related phenotypic modules in XYY showed stronger relationships with the adaptive functioning module than they did with each other.

\section{Discussion}

To date, the behavioral phenotype of XYY has been collectively defined using partially overlapping measures in different cohorts. The current study reinforces several key findings of these prior studies within a previously unreported single-center cohort, including delays in motor and language development (cf. [10]), wide-ranging IQ with a downward-shifted distribution and relative deficits in verbal scores (cf. [4]), and reduced adaptive functioning (cf. [8]). Our evaluations also revealed relatively low academic achievement scores, with the majority of participants meeting criteria for at least one SLD. However, we note that academic achievement scores were relatively similar between the pre- vs. postnatally diagnosed groups, despite significant group differences in a range of cognitive and behavioral domains. We speculate that this dissociation might arise due to group differences in (i) unmeasured factors (in addition to variables tested here like cognition), that contribute to academic achievement, and/or (ii) academic remediation [33, 34]. Access to dense and homogenous phenotypic data allowed us to resolve a gradient of vulnerability across 
Table 2 Sensitivity and specificity of ASD measures

\begin{tabular}{|c|c|c|c|c|c|}
\hline Stratification & $\operatorname{ASD}(n)$ & Non-ASD $(n)$ & AUC (95\% Cl) & Sensitivity (95\% Cl) & Specificity $(95 \%$ CI) \\
\hline \multicolumn{6}{|l|}{ ADI-R } \\
\hline Full sample & 9 & 54 & $0.97(0.93-1)$ & $1.00(0.66-1.00)$ & $0.87(0.75-0.95)$ \\
\hline \multicolumn{6}{|c|}{ CBCL externalizing } \\
\hline$<64$ & 6 & 33 & $0.98(0.96-1)$ & $1.00(0.54-1.00)$ & $0.91(0.76-0.98)$ \\
\hline$\geq 64$ & 3 & 19 & $0.95(0.84-1)$ & $1.00(0.29-1.00)$ & $0.84(0.60-0.97)$ \\
\hline \multicolumn{6}{|c|}{ CBCL internalizing } \\
\hline$<64$ & 4 & 32 & $1(1-1)$ & $1.00(0.40-1.00)$ & $0.91(0.75-0.98)$ \\
\hline$\geq 64$ & 5 & 20 & $0.93(0.82-1)$ & $1.00(0.48-1.00)$ & $0.85(0.62-0.97)$ \\
\hline \multicolumn{6}{|l|}{ ADOS-2 } \\
\hline Full sample & 9 & 53 & $0.97(0.93-1)$ & $0.89(0.52-1.00)$ & $0.98(0.90-1.00)$ \\
\hline \multicolumn{6}{|c|}{ CBCL externalizing } \\
\hline$<64$ & 6 & 32 & $0.99(0.96-1)$ & $1.00(0.54-1.00)$ & $1.00(0.89-1.00)$ \\
\hline$\geq 64$ & 3 & 19 & $0.92(0.79-1)$ & $0.67(0.09-0.99)$ & $0.90(0.74-1.00)$ \\
\hline \multicolumn{6}{|c|}{ CBCL internalizing } \\
\hline$<64$ & 4 & 31 & $1(1-1)$ & $1.00(0.40-1.00)$ & $1.00(0.89-1.00)$ \\
\hline$\geq 64$ & 5 & 20 & $0.91(0.79-1)$ & $0.80(0.28-0.99)$ & $0.91(0.75-1.00)$ \\
\hline \multicolumn{6}{|l|}{ SRS-2 } \\
\hline Full sample & 9 & 52 & $0.85(0.75-0.96)$ & $1.00(0.66-1.00)$ & $0.59(0.45-0.73)$ \\
\hline \multicolumn{6}{|c|}{ CBCL externalizing } \\
\hline$<64$ & 6 & 33 & $0.92(0.82-1)$ & $1.00(0.54-1.00)$ & $0.69(0.51-0.84)$ \\
\hline$\geq 64$ & 3 & 19 & $0.72(0.51-0.93)$ & $1.00(0.29-1.00)$ & $0.42(0.21-0.67)$ \\
\hline \multicolumn{6}{|c|}{ CBCL internalizing } \\
\hline$<64$ & 4 & 32 & $0.96(0.89-1)$ & $1.00(0.40-1.00)$ & $0.75(0.57-0.89)$ \\
\hline$\geq 64$ & 5 & 20 & $0.68(0.44-0.91)$ & $1.00(0.48-1.00)$ & $0.36(0.15-0.59)$ \\
\hline \multicolumn{6}{|l|}{ SCQ } \\
\hline Full sample & 9 & 52 & $0.85(0.73-0.96)$ & $0.78(0.40-0.97)$ & $0.73(0.61-0.85)$ \\
\hline \multicolumn{6}{|c|}{ CBCL externalizing } \\
\hline$<64$ & 6 & 33 & $0.86(0.72-1.00)$ & $0.66(0.22-0.95)$ & $0.76(0.58-0.89)$ \\
\hline$\geq 64$ & 3 & 19 & $0.80(0.60-1.00)$ & $1.00(0.29-1.00)$ & $0.68(0.43-0.87)$ \\
\hline \multicolumn{6}{|c|}{ CBCL internalizing } \\
\hline$<64$ & 4 & 32 & $0.90(0.80-1.00)$ & $1.00(0.40-1.00)$ & $0.81(0.64-0.93)$ \\
\hline$\geq 64$ & 5 & 20 & $0.77(0.56-0.98)$ & $0.60(0.15-0.95)$ & $0.60(0.36-0.81)$ \\
\hline
\end{tabular}

ASD and non-ASD DSM-5 diagnosis. AUC (area under the curve), sensitivity, and specificity refer to the comparison of the given test cut-off to the DSM-5 diagnosis

different dimensions of neurodevelopment, ranging from greatest impairment in average adaptive socialization skills to least impairment in average NVIQ. These measures also helped to better-resolve potential ascertainment bias effects: we replicated the prior finding of below-average IQ among prenatally vs. postnatally diagnosed individuals with XYY (cf. [5]), and further detect statistically significant time of diagnosis effects for VIQ, processing speed, adaptive social functioning, and internalizing symptoms. This effort represents a step towards securing more accurate estimates of the penetrance of XYY for a range of neurodevelopmental issues, with the ultimate goal of informing the provision of genetic counseling in affected pregnancies.
Understanding the nature of the relationship between $\mathrm{XYY}$ and ASD is not only important for clinical understanding of XYY, but also for evaluating the notion that Y-chromosome dosage effects could be relevant to the male bias in ASD prevalence. While one-third of this sample had a community ASD diagnosis, only a subset of these participants met gold standard criteria for a research-based diagnosis. The rate of ASD in this sample (14\%), according to DSM-5 criteria applied after gold standard diagnostic instruments were given to all study participants, is lower than reported in earlier studies [7, 14], but still represents a six-fold increase above the baseline rate of ASD (2.38\%) in males [33]. Discrepancies between previous 


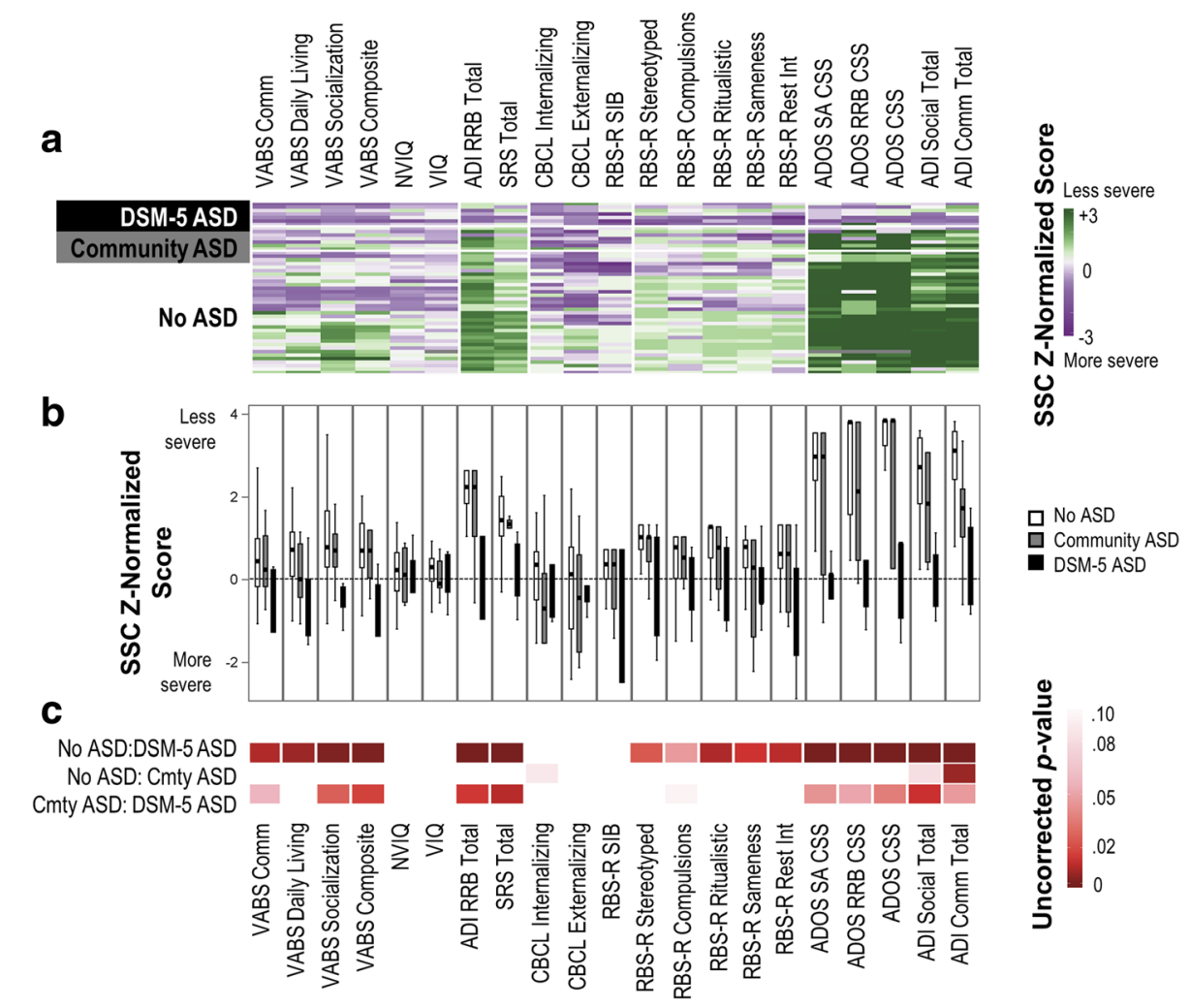

Fig. 2 Phenotypic profiles of XYY participants younger than 18 years $(n=49)$. a $Z$ normalized scores for each participant in the XYY sample ( $Y$ axis), using the means and standard deviations from the Simons Simplex Collection (SSC). b Boxplots of $Z$ normalized scores by diagnostic status. $\mathbf{c} p$ values for pairwise comparisons

community diagnoses and current research-based diagnoses are illustrative of the growing literature regarding variability in diagnostic stability in ASD (see [36] for a review) and may be attributed to a variety of factors, including change in diagnostic nosology from DSM-IV-TR to DSM-5 (e.g., lack of total overlap between previous diagnosis of PDD-NOS and current ASD diagnosis); clinical instability of diagnoses based on type of previous diagnosis, clinical setting, and, diagnosis process [37]. A larger XYY ASD cohort is required to achieve adequate statistical power for formal comparison of the broader neurodevelopmental profile of ASD within XYY, but here we did not see a unique profile in comparison to idiopathic ASD. An important goal for future work will be determining whether the observed rate of ASD in XYY is significantly elevated beyond that seen in other neurogenetic disorders with comparable levels of general developmental difficulties.

Measures of ASD symptoms, especially parent reports and/or screeners, are vulnerable to the confounding effects of impaired cognitive ability, high rates of problem behavior, and/or level of suspicion of ASD [34-36]. Indeed, in this study, we found that the sensitivity of all ASD assessment instruments was excellent, but the specificity of the dimensional questionnaire-based measures of ASD-unlike interviewer-interpreted measurement with the ADI-R and ADOS-2-was especially affected by internalizing and externalizing problems. This reflects a general psychometric challenge in ASD assessment, rather than XYY-specific phenomena [37]. Still, the limitations of these instruments in populations with high rates of problem behaviors, like XYY, must be recognized and mitigated with thorough clinical assessment and judgment. These clinical distinctions are paramount, given that they confer specific treatment priorities, which may necessitate the need for a multidisciplinary clinic type evaluation that can disentangle how these multiple symptom presentations impact functioning.

Finally, our integrative analysis suggested three separable phenotypic modules, which may provide more refined cognitive/behavioral targets for future genetic and neurobiological studies. The agnostic nature of these analyses allows for potential discovery of feature cluster that derive from unexpected co-segregations or dissociations of conventional symptom domains. For example, the "yellow" cluster in Fig. 3 combines developmental milestones with measures of adaptive behavior, while it splits features of internalizing disorders away from symptoms of externalizing psychopathology. Graph-theoretical treatment of 


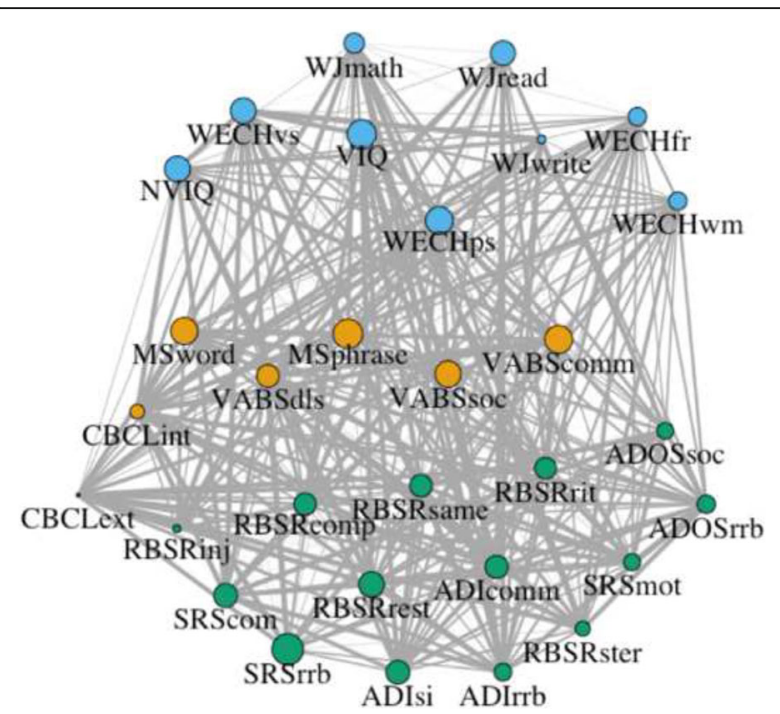

Fig. 3 Network representation of phenotypic modules in XYY $(N=64)$. Nodes are phenotypic variables; color distinguishes reproducible clusters: cognitive ability/academic achievement (blue), adaptive functioning/ psychopathology (yellow), and ASD-related features (green). Size indices the mean pairwise correlation between the variable and all others. Thicker edges show increased consistency of co-clustering based on bootstrapped analysis. The force-directed Fruchterman-Reingold Algorithm was used, such that further distance between nodes indicates weaker inter-relationships

clinical features also facilitates future integration with graph-theoretical analyses of neuroimaging data-potentially allowing for detection of linked modules of altered brain and behavior in XYY syndrome.

\section{Limitations and future directions}

Although we quantitatively measured a wide variety of neurodevelopmental features, with a specific focus on ASD symptoms and diagnosis, the range of behaviors that can be assessed in a single study are limited by practical considerations (e.g., time constraints), primary use of caregiver questionnaires vs. inclusion of schoolbased measures, and availability of validated instruments. Nevertheless, access to a greater diversity of scales in future studies-including different assessment methods (e.g., caregiver vs. teacher vs. performance based) for the same developmental domain-would help to further characterize neurodevelopmental features of XYY syndrome, and more firmly resolve dissociable subsets among these features.

While our comparison of profiles with a similarly characterized ASD cohort provided some context, differential ascertainment methods between these groups may limit comparability. Relatedly, the wide age range and cross-sectional data limit our understanding of how time itself impacts the course and severity of symptom profiles described. It was also not practical to have the evaluators be masked to all information regarding previously established genetic diagnoses throughout their assessments, but this would have been ideal. We attempted to address the ascertainment bias that exists in many XYY studies by exploring differences in timing of XYY diagnosis, which may not have completely resolved the possibility of bias. However, definitive control of such biases will require new, large-scale genetic testing of population-based birth cohorts. In the meantime, studies of infant and toddler development among prenatally diagnosed groups with sex chromosome aneuploidy will be transformative.

Given the frequency of XYY and the limited (albeit substantial) cohort evaluated, this study may offer some cautiously provided guidance regarding the types of assessments and intervention that may be useful to support and improve outcomes for individuals identified with XYY, although none of these proposals should be considered unique to XYY. Based on the variability in impairments observed in this sample, it is recommended that evaluations for this population include components assessing cognitive, adaptive, and academic skills along with evaluation of behavioral problems to provide a comprehensive assessment of potentially impacted domains. Additionally, if screening indicates a referral for an ASD assessment, the use of a team evaluation comprised of autism experts using gold standard measures of ASD symptoms is recommended to increase the likelihood of the provision of accurate and stable diagnoses. Early intervention along with the later academic interventions and the potential need for targeted support in adaptive skill development should be considered.

An important, yet challenging goal for future research will be specifying the genomic mechanisms through which carriage of a supernumerary Y-chromosome can influence human neurodevelopment. These mechanisms may involve altered expression of dosage-sensitive Y-linked gametologs such as NLGN4Y [38] that are expressed in the brain [39] and have been argued to influence neurodevelopmental traits [16]. However, there are currently no means of conducting definitive tests for such hypotheses given ethical and practical obstacles to controlled experimental manipulation of brain gene expression in humans. Finally, we noted considerable variability in the phenotypic presentation of XYY, and future investigation should also seek genetic and environmental factors that can account for these inter-individual differences in expressivity among males carrying an extra Y-chromosome.

\section{Conclusion}

Males with XYY have variable neurodevelopmental presentation, but on average, have lower cognitive, adaptive, language, and academic skills than the general 
population. Though still elevated relative to the general population, the rate of ASD in XYY may be lower than suggested by earlier studies. However, there is some evidence for variable ascertainment bias effects across different facets of the neurodevelopmental phenotype in XYY that can only be addressed in population-based birth cohort studies.

\begin{abstract}
Abbreviations
ADI-R: Autism Diagnostic Interview, Revised; ADOS-2: Autism Diagnostic Observation Schedule, second edition; ASD: Autism spectrum disorder; CBCL: Child Behavior Checklist; FSIQ: Full Scale Intelligence Quotient; IQ: Intelligence quotient; NVIQ: Nonverbal Intelligence Quotient; RBS-R: Repetitive Behavior Scale, Revised; SCQ: Social Communication Questionnaire; SRS-2: Social Responsiveness Scale, second edition; SSC: Simons Simplex Collection; VABS-II: Vineland Adaptive Behavior Scale, second edition; VIQ: Verbal Intelligence Quotient; WJ-IV: Woodcock-Johnson IV
\end{abstract}

\section{Acknowledgements}

We thank all the young people and families who took part in this research. We are grateful to the SSC families and principal investigators (Beaudet, Bernier, Constantino, Cook, Fombonne, Geschwind, Goin-Kochel, Hanson, Grice, Klin, Ledbetter, Lord, Martin, Martin, Maxim, Miles, Ousley, Pelphrey, Peterson, Piggot, Saulnier, State, Stone, Sutcliffe, Walsh, Warren, Wijsman).

\section{Funding}

This work was supported by the Intramural Research Program of the National Institute of Mental Health, (Protocol 89-M-S009, NCT00001246, ZIAMH002794).

\section{Availability of data and materials}

The Simons Simplex Collection data are available from the Simons Simplex Collection repository. Approved researchers can obtain these data by applying at https://base.sfari.org. The XYY data are registered with ClinicalTrials.gov under the name "89-M-0006: Brain Imaging of Childhood Onset Psychiatric Disorders, Endocrine Disorders and Healthy Controls."

\section{Authors' contributions}

$\mathrm{LJ}$ contributed to the design of the study, collected and analyzed the data, and drafted the manuscript. CF contributed to the design of the study, analyzed the data, and revised the manuscript. CC, LH, AF, CM, AX, $L C, B S, J S, J B$, and ET contributed to the data collection and analysis, and revision of the manuscript. AT and $A A$ contributed to the design of the study, analyzed the data, and revised the manuscript. All authors read and approved the final manuscript.

\section{Ethics approval and consent to participate}

Informed consent and assent was obtained from all participants and their parents; all study procedures were approved by a National Institutes of Health Institutional Review Board.

\section{Consent for publication}

Not applicable.

\section{Competing interests}

The authors declare that they have no competing interests.

\section{Publisher's Note}

Springer Nature remains neutral with regard to jurisdictional claims in published maps and institutional affiliations.

\section{Author details}

'Office of the Clinical Director, National Institute of Mental Health, National Institute of Health, Bethesda, MD, USA. '2Developmental Neurogenomics Unit, Human Genetics Branch, National Institute of Mental Health, National Institutes of Health, Bethesda, MD, USA.
Received: 23 April 2018 Accepted: 20 September 2018

Published online: 22 October 2018

\section{References}

1. Nielsen J, Wohlert M. Chromosome abnormalities found among 34,910 newborn children: results from a 13-year incidence study in Arhus, Denmark. Hum Genet. 1991;87:81-3.

2. Jacobs PA, Brunton M, Melville MM, Brittain RP, McClemont WF. Aggressive behavior, mental sub-normality and the XYY male. Nature. 1965;208:1351-2.

3. Stochholm K, Bojesen A, Jensen AS, Juul S, Gravholt CH. Criminality in men with Klinefelter's syndrome and XYY syndrome: a cohort study. BMJ Open. 2012;2:e000650.

4. Leggett V, Jacobs P, Nation K, Scerif G, Bishop DV. Neurocognitive outcomes of individuals with a sex chromosome trisomy: $X X X, X Y Y$, or $X X Y$ : a systematic review. Dev Med Child Neurol. 2010;52:119-29.

5. Bardsley MZ, Kowal K, Levy C, Gosek A, Ayari N, Tartaglia N, Lahlou N, Winder B, Grimes S, Ross JL. 47,XYY syndrome: clinical phenotype and timing of ascertainment. J Pediatr. 2013;163:1085-94.

6. Bishop DVM, Jacobs PA, Lachlan K, Wellesley D, Barnicoat A, Boyd PA, Fryer A, Middlemiss P, Smithson S, Metcalfe K, et al. Autism, language and communication in children with sex chromosome trisomies. Arch Dis Child. 2011;96:954-9.

7. Tartaglia N, Wilson R, Miller JS, Rafalko J, Cordeiro L, Davis S, Hessl D, Ross J. Autism spectrum disorder in males with sex chromosome aneuploidy: XXY/ Klinefelter syndrome, XYY, and XXYY. J Dev Behav Pediatr. 2017;38:197-207.

8. Tartaglia N, Ayari N, Hutaff-Lee C, Boada R. Attention-deficit hyperactivity disorder symptoms in children and adolescents with sex chromosome aneuploidy: XXY, XXX, XYY, and XXYY. J Dev Behav Pediatr. 2012;33:309-18.

9. Hong DS, Reiss AL. Cognitive and neurological aspects of sex chromosome aneuploidies. Lancet Neurol. 2014;13:306-18.

10. Lalatta F, Folliero E, Cavallari U, Di Segni M, Gentilin B, Fogliani R, Quagliarini D, Vizziello P, Monti F, Gargantini L. Early manifestations in a cohort of children prenatally diagnosed with 47,XYY. Role of multidisciplinary counseling for parental guidance and prevention of aggressive behavior. Ital J Pediatr. 2012;38:52.

11. Geerts M, Steyaert J, Fryns JP. The XYY syndrome: a follow-up study on 38 boys. Genet Couns. 2003;14:267-79.

12. Ratcliffe S. Long-term outcome in children of sex chromosome abnormalities. Arch Dis Child. 1999;80:192-5.

13. Ross $J$, Zeger MP, Kushner $H$, Zinn AR, Roeltgen DP. An extra X or $Y$ chromosome: contrasting the cognitive and motor phenotypes in childhood in boys with 47,XYY syndrome or 47,XXY Klinefelter syndrome. Dev Dis Res Rev. 2009;15:309-17.

14. Margari L, Lamanna AL, Craig F, Simone M, Gentile M. Autism spectrum disorders in XYY syndrome: two new cases and systematic review of the literature. Eur J Pediatr. 2014;173:277-83.

15. Lee NR, Wallace GL, Adeyemi El, Lopez KC, Blumenthal JD, Clasen LS, Giedd $J N$. Dosage effects of $X$ and $Y$ chromosomes on language and social functioning in children with supernumerary sex chromosome aneuploidies: implications for idiopathic language impairment and autism spectrum disorders. J Child Psychol Psychiatry Allied Discip. 2012;53:1072-81.

16. Ross JL, Tartaglia N, Merry DE, Dalva M, Zinn AR. Behavioral phenotypes in males with XYY and possible role of increased NLGN4Y expression in autism features. Genes Brain Behav. 2015;14:137-44.

17. Werling DM, Geschwind DH. Sex differences in autism spectrum disorders. Curr Opin Neurol. 2013;26:146-53.

18. Stochholm K, Juul S, Gravholt C. Diagnosis and mortality in 47,XYY persons: a registry study. Orphanet J Rare Dis. 2010;5:15.

19. Rutter M, LeCouteur A, Lord C. Autism diagnostic interview-revised (ADI-R). Los Angeles: Western psychological services; 2003.

20. Sparrow SS, Cicchetti DV, Balla DA. Vineland adaptive behavior scales. 2nd ed. Circle Pines: AGS Publishing; 2005.

21. Lord C, Rutter M, PC DL, Risi S, Gotham K, Bishop SL. Autism Diagnostic Observation Schedule, Second Edition (ADOS-2) Manual (Part 1): Modules 1-4. Torrance: Western Psychological Services; 2012.

22. American Psychiatric Association. diagnostic and statistical manual of mental disorders. 5th ed. Arlington: American Psychiatric Publishing; 2013.

23. Constantino JN, Grubar JC. The social responsiveness scale manual, second edition (SRS-2). Los Angeles: Western Psychoological Association; 2012.

24. Rutter M, Bailey A, Lord C. The social communication questionnaire. Los Angeles: Western Psychological Services; 2003. 
25. Lam KS, Aman MG. The repetitive behavior scale-revised: independent validation in individuals with autism spectrum disorders. J Autism Dev Disord. 2007;37:855-66

26. Maechler M, Rousseeuw P, Struyf A, Hubert M, Hornik K: Cluster: Cluster Analysis Basics and Extensions. R packages version 2.0.6. 2017.

27. Csardi G, Nepusz T. The igraph software package for complex network research. InterJ Complex Syst. 2006;1695:1-9.

28. R Core Team. R: a language and environment for statistical computing. Vienna: R Foundation for statistical Computing; 2014

29. Barter RL, Yu B: Superheat: an R package for creating beautiful and extendable heatmaps for visualizing complex data. arXiv preprint arXiv: 1512015242015

30. Wilke C: Ggridges: Ridgeline plots in 'ggplot2'. R package version 0.4.1; 2017.

31. Rescorla L. The language development SurveyA screening tool for delayed language in toddlers. J Speech Hear Dis. 1989:54:587-99.

32. Who Multicentre Growth Reference Study G, de Onis M. WHO motor development study: windows of achievement for six gross motor development milestones. Acta Paediatr. 2006;95:86-95.

33. Christensen DL, Baio J, Van Naarden Braun K, Bilder D, Charles J, Constantino JN, Daniels J, Durkin MS, Fitzgerald RT, Kurzius-Spencer M, et al. Prevalence and characteristics of autism spectrum disorder among children aged 8 years--autism and developmental disabilities monitoring network, 11 sites, United States, 2012. MMWR Surveill Summ. 2016;65:1-23.

34. Havdahl KA, Hus Bal V, Huerta M, Pickles A, Oyen AS, Stoltenberg C, Lord C, Bishop SL. Multidimensional influences on autism symptom measures: implications for use in etiological research. J Am Acad Child and Adolesc Psychiatry. 2016;55:1054-1063.e1053.

35. Hus V, Bishop S, Gotham K, Huerta M, Lord C. Factors influencing scores on the social responsiveness scale. J Child Psychol Psychiatry. 2013;54:216-24.

36. Kaat AJ, Farmer C. Commentary: lingering questions about the social responsiveness scale short form. A commentary on Sturm et al.(2017). J Child Psychol Psychiatry. 2017;58:1062-4.

37. Charman T, Gotham K. Measurement issues: screening and diagnostic instruments for autism spectrum disorders - lessons from research and practice. Child Adolesc Mental Health. 2013;18:52-63.

38. Raznahan A, Parikshak NN, Chandran V, Blumenthal JD, Clasen LS, Alexander-Bloch AF, Zinn AR, Wangsa D, Wise J, Murphy DGM, Bolton PF, Ried T, Ross J, Giedd JN, Geschwind DH. Sex-chromosome dosage effects on gene expression in humans. Proc Natl Acad Sci. 2018;115(28):7398-403.

39. Bellot DW, Hughes JF, Skalesty H, Brown LG, Pyntikova T, Cho TJ, Koutseva N, Zaghlul S, Graves T, Rock S, Kremitzki C, Fulton RS, Dugan S, Ding Y, Morton D, Khan Z, Lewis L, Buhay C, Wang Q, Watt J, Holder M, Lee S, Nazareth L, Alföldi J, Rozen S, Muzny DM, Warren WC, Gibbs RA, Wilson RK, Page DC. Mammalian Y chromosomes retain widely expressed dosagesensitive regulators. Nature. 2014;508(7497):494-9.

Ready to submit your research? Choose BMC and benefit from:

- fast, convenient online submission

- thorough peer review by experienced researchers in your field

- rapid publication on acceptance

- support for research data, including large and complex data types

- gold Open Access which fosters wider collaboration and increased citations

- maximum visibility for your research: over $100 \mathrm{M}$ website views per year

At $\mathrm{BMC}$, research is always in progress.

Learn more biomedcentral.com/submissions 